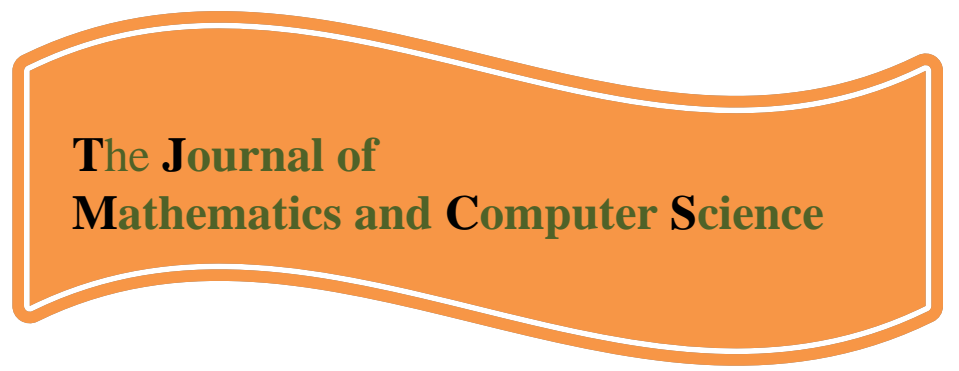

Available online at

http://www.TJMCS.com

The Journal of Mathematics and Computer Science Vol .2 No.3 (2011) 413-416

\title{
New exact-analytical solutions for the mKdV equation
}

\author{
S. R. Mousavian ${ }^{1,}{ }^{*}$, H. Jafari ${ }^{2}$, C. M. Khalique ${ }^{3}$ and S. A. Karimi \\ ${ }^{1}$ Department of Mathematics, Islamic Azad University, Jouybar Branch, Jouybar, Mazandaran, Iran \\ ${ }^{2}$ Department of Mathematics, University of Mazandaran, Babolsar, Mazandaran, Iran \\ 3International Institute for Symmetry Analysis and Mathematical Modelling, Department of Mathematical Sciences, \\ North-West University, Mafikeng Campus, Mmabatho 2735, South Africa \\ jafari@umz.ac.ir
}

Received: August 2010, Revised: November 2010

Online Publication: January 2011

\begin{abstract}
In this paper, the sine-cosine, the extended tanh method has been used to obtain solutions of the modified $\mathrm{KdV}(\mathrm{mKdV})$ equation. New periodic solutions are formally derived.
\end{abstract}

Keywords: The nonlinear evolutions equations, the sine-cosine method, the extended tanh method, the modified KdV equation.

\section{Introduction}

The investigation of exact traveling wave solutions to nonlinear evolutions equations plays an important role in the study of nonlinear physical phenomena. Applications of the results obtained for the mKdV equation in this paper is to description of ion-acoustic waves in an unmagnetized plasma.

\footnotetext{
$1,{ }^{*}$ Corresponding author

${ }^{2}$ Assistant Professor

${ }^{3}$ Assistant Professor
} 
The aim of the present paper is to extend the sine-cosine and the extended tanh methods to finding new exact solutions for $\mathrm{mKdV}$ equation where is expressed as follows[7]

$$
u_{t}+6 s u^{2} u_{x}-u_{x x x}=0, \quad s= \pm 1
$$

\section{Analysis of method}

For the sine-cosine and the extended tanh methods, we first unite the independent variable $\mathrm{x}$ and $t$ into one wave variable $\xi=x-c t$, to carry out a $P D E$ in two independent variables

$$
P\left(u, u_{x}, u_{t}, u_{x x}, \ldots\right)=0
$$

into an $O D E$

$$
Q\left(u, u^{\prime}, u^{\prime \prime}, u^{\prime \prime \prime}, \ldots\right) \text {. }
$$

Eq.(3) is then integrated as long as all terms contain derivative. Usually the integration constants are considered to be zeros in view of the localized solutions.

\subsection{The sine-cosine method}

For this method the solutions of the reduced $O D E$ equation can be expressed in the form

$$
u(x, t)=\left\{\begin{array}{lc}
\lambda \cos ^{\beta}(\mu \xi) & |\mu \xi| \leq \frac{\pi}{2} \\
0 & \text { else }
\end{array}\right.
$$

Where $\lambda, \mu, \beta$ are parameters that will be determined. $[2,3,4,5]$

Substituting the cosine assumption and their derivatives into the reduce $O D E$ gives a trigonometric equation of $\cos ^{k}(\mu \xi)$ or $\sin ^{k}(\mu \xi)$ terms. The parameters are then determined by first balancing the exponents of each pair of cosine determine $\mathrm{k}$. We next collect all coefficients of the same power in $\cos ^{k}(\mu \xi)$ or $\sin ^{k}(\mu \xi)$ where these coefficients have to vanish. This gives a system of algebraic equations among the unknowns $c, \lambda, \mu, \xi$ that will be determined.

\subsection{The extended tanh method}

The extended tanh method introduces a new independent variable $Y=\tanh (\mu \xi)$, that leads to the changes of derivatives:

$$
\frac{d}{d \xi}=\mu\left(1-Y^{2}\right) \frac{d}{d Y} \quad, \quad \frac{d^{2}}{d \xi^{2}}=\mu^{2}\left(1-Y^{2}\right)\left(-2 \mathrm{Y} \frac{\mathrm{d}}{\mathrm{dY}}+\left(1-Y^{2}\right) \frac{d^{2}}{d Y^{2}}\right) .
$$

The solutions for the extended tanh method can be proposed as a finite power series in $Y$ in the form

$$
u(\mu \xi)=S(Y)=\sum_{k=0}^{M} a_{k} Y^{k}+\sum_{k=1}^{M} b_{k} Y^{-k}
$$

Where the parameter $M$ is a positive, in most cases, that will be determined. To determine the parameter $M$, we usually balance the linear terms of highest order in the resulting equation with the highest order nonlinear terms. We then collect all coefficients of powers of $Y$ in the resulting equation where these coefficients have to vanish. This will give a system of algebraic equations involving the parameters $a_{k}(k=0, \ldots, M), b_{k}(k=1, \ldots, M), \mu, \xi$, and $c$. Having determined these parameters we obtain an analytic solution $u(x, t)$ in a closed form. $[1,6]$

\section{Application of methods}

\subsection{Using the sine-cosine method}

Substituting $u(x, t)=u(\xi)$, where the wave variable is $\xi=x-c t$, carries out the mKdV equation(1) to the $O D E$

$$
-c u^{\prime}+6 s u^{2} u^{\prime}-u^{\prime \prime \prime}=0
$$


Once integrating (7), setting the constant of integrating to zero, we obtain

$$
-c u+2 s u^{3}-u^{\prime \prime}=0 \text {. }
$$

Substituting cosine anstaz (4) into (8) gives

$$
-c \lambda \cos ^{\beta}(\mu \xi)+2 s \lambda^{3} \cos ^{3 \beta}(\mu \xi)+\lambda \mu^{2} \beta^{2} \cos ^{\beta}(\mu \xi)+\lambda \mu^{2} \beta(\beta-1) \cos ^{\beta-2}(\mu \xi)=0 \text {. }
$$

Equating the exponents of the second and the last cosine functions in above equation, collecting coefficients of each pair of cosine functions of like exponents, and setting it equal to zero, we obtain the following system of algebraic equations:

$$
\left\{\begin{array}{l}
\beta-1 \neq 0, \\
\beta-2=3 \beta, \\
c=\mu^{2} \beta^{2}, \\
2 s \lambda^{3}=\lambda \mu^{2} \beta(\beta-1) .
\end{array}\right.
$$

so that this gives

$$
\left\{\begin{array}{l}
\beta=-1, \\
c=\mu^{2}, \\
\lambda=\sqrt{s} \mu .
\end{array}\right.
$$

and $\mu$ is any real number.

Substituting (11) into (4), we find the following solution for modified KdV equation:

$$
u(x, t)= \begin{cases}\sqrt{s} \mu \sec \left(x-\mu^{2} t\right) & |\mu \xi| \leq \frac{\pi}{2} \\ 0 & \text { else. }\end{cases}
$$

\subsection{Using the extended tanh method}

As shown before, the $\mathrm{mKdV}$ equation(1) can be transformed to the $O D E$

$$
-c u+2 s u^{3}-u^{\prime \prime}=0 \text {. }
$$

Balancing $u^{\prime \prime}$ with $u^{3}$ gives $M+2=3 M$, so that $M=1$, therefore the extended tanh method assumes that finite expansion

$$
u(\mu \xi)=S(Y)=a_{0}+a_{1} Y+b_{1} Y^{-1}
$$

where $Y=\tanh (\mu \xi)$. Substituting (13) into (12) and using (5), collecting the coefficients of $Y^{j}(-3 \leq j \leq 3)$ and equating this coefficients to zero and solving the system of algebraic for $a_{0}, a_{1}, b_{1}, \mu$ and $c$ we find the following set of

$$
\left\{\begin{array}{l}
a_{0}=0, \\
a_{1}=0 \\
b_{1}=\sqrt{s} \mu, \\
c=2 \mu^{2} .
\end{array}\right.
$$

and $\mu$ is any real number. Substituting (14), into (6) gives

$$
u(x, t)=\sqrt{s} \mu \tanh ^{-1}\left\{\mu\left(x-2 \mu^{2} t\right)\right\} .
$$

\section{Conclusions}

The sine-cosine, the extended tanh methods were used to investigate nonlinear mKdV equation. The work emphasized our belief the two methods are powerful technique to handle nonlinear 
digestive equation, hence these methods can be used in a wider context. The validity of these methods has been tested by applying to modified KdV equation. Finally it is a promising and powerful method for other nonlinear equations in mathematical physics.

\section{References}

[1] A. M. Wazwaz, The extended tenh method for Zhakharov-Kkuznetsov (ZK) equation, the modified ZK equation, andits generalized forms. Communications in Nonlinear Science and Numerical Simulation., 13(6) 2008, 1039-1047.

[2] A. M. Wazwaz, Partial Differential Equations: Methods and Applications, Balkema, Rotterdam, 2002.

[3] A. M. Wazwaz, Dustinct variants of the KdV equation with compact and noncompact structures, Appl. Math. Comput., 150 (2004) 365-377.

[4] A. M. Wazwaz, Variants of the generalized KdV eauation with compact and noncompact structures, Comput. Math. Appl., 47 (2004) 583-591.

[5] A. M. Wazwaz, An analytical study of the compactons structures in a class of nonlinear dispersive equation. Math Comput Simulation., 63(1) (2003) 35-44.

[6] A. M. Wazwaz, A computational approachto soliton solutions of the Kadomtsev-Petviashili equation. Comput. Math. Appl.,123(2) (2001) 205-217.

[7] Inc. Mustafa, Numerical simulation of KdV and mKdV equations with initial conditions by the variational iteration method, Choas, Solitons and Fractals., Accepted 20 April 2006. 